\title{
Genome-wide transcriptional analysis of T cell activation reveals differential gene expression associated with psoriasis
}

\author{
Nuria Palau', Antonio Julià', Carlos Ferrándiz², Lluís Puig ${ }^{3}$, Eduardo Fonseca ${ }^{4}$ Emilia Fernández ${ }^{5}$, \\ María López-Lasanta', Raül Tortosa ${ }^{1}$ and Sara Marsal ${ }^{1 *}$
}

\begin{abstract}
Background: Psoriasis is a chronic autoimmune disease in which T cells have a predominant role in initiating and perpetuating the chronic inflammation in skin. However, the mechanisms that regulate $T$ cell activation in psoriasis are still incompletely understood. The objective of the present study was to characterize the main genetic pathways associated with $T$ cell activation in psoriasis.

Results: Gene expression profiles from in vitro activated T cells were obtained from 17 psoriasis patients and 7 healthy controls using Illumina HT-12 v4 microarrays. From a total of 47,321 analyzed transcripts, 42 genes were found to be differentially expressed between psoriasis and controls (FDR p-value $<0.1$, absolute fold-change $>1.2$ ). Using an independent cohort of 8 patients and 8 healthy controls we validated the overexpression of SPATS2L ( $p$-value $=0.0009)$ and KLF6 ( $p$-value $=0.0012)$ genes in activated T cells from psoriasis patients. Using weighted correlation analysis we identified SPATS2L and KLF6 coexpression networks, which were also significantly associated with psoriasis ( $p$-value $<0.05$ ). Gene Ontology analysis allowed the identification of several biological processes associated with each coexpression network. Finally, using Gene Set Enrichment Analysis over the global T cell transcriptome we also found additional genetic pathways strongly associated with psoriasis ( $p$-value $<0.0001$ ).

Conclusions: This study has identified two new genes, SPATS2L and KLF6, strongly associated with T cell activation in psoriasis. Functional analyses of the gene expression profiles also revealed new biological processes and genetic pathways associated with psoriasis. The results of this study provide an important insight into the biology of this common chronic inflammatory disease.
\end{abstract}

Keywords: Psoriasis, T cell, Gene expression, Microarray, Genetic pathway, Gene network

\section{Background}

Psoriasis is a chronic T-cell-mediated inflammatory disease that affects approximately $2 \%$ of the world population [1]. Histologically, it is characterized by the infiltration of active immune cells into the skin, the hyperplasia of blood vessels and the hyperproliferation of keratinocytes. The leukocyte infiltrate in psoriasis lesions is composed mainly by $\mathrm{T}$ cells, predominantly CD4 + -type cells but also CD8 + -type T cells [2]. T cells are known to expand and migrate into the epidermis

\footnotetext{
* Correspondence: sara.marsal@vhir.org

${ }^{1}$ Rheumatology Research Group, Vall d'Hebron Research Institute, Barcelona 08035, Spain

Full list of author information is available at the end of the article
}

before the onset of the first epidermal changes, and steadily continue to do so, promoting and perpetuating the disease [3].

The central role of $\mathrm{T}$ cells in psoriasis pathogenesis was first evidenced in the late 70s, when the T cell suppressor cyclosporine A was discovered as an effective treatment for psoriasis inflammation [4]. In the 90's elegant studies using skin xenografts of with non-lesional skin from patients with psoriasis showed the conversion to psoriatic plaques when mice were injected with preactivated $\mathrm{T}$ cells from the same patient [5]. Since then, a substantial amount evidence supporting the key role for T cells, mainly type $1 \mathrm{~T}$ helper subtype (Th1), has been accumulated [6-11]. More recently, IL17 and IL22

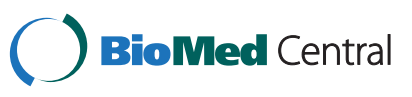


producing $\mathrm{T}$ lymphocytes (Th17 and Th22, respective) have been characterized as critical mediators of psoriasis pathogenesis [12-14]. However, the triggering events and deregulated pathways that lead to the aberrant $\mathrm{T}$ cell activation in psoriasis patients are still poorly defined. In the present study we aimed to increase the knowledge on this particular aspect of psoriasis etiology by characterizing the $\mathrm{T}$ cell gene expression profile associated with psoriasis during $\mathrm{T}$ cell activation.

To date, psoriasis has been investigated by a number of whole genome gene expression studies. These have been mainly conducted with skin biopsy samples, through the comparison of lesional and non-lesional skin. Gene expression analysis in biopsies from skin lesions is of great interest in psoriasis and has produced several important findings [15-21]. However, one limitation of this approach is that the mixture of the different cell types that coexist in the skin tissue is likely to mask relevant gene expression patterns. Specially, if the cell type of interest is not the predominant component of the total tissue extract, as in the case of infiltrating $\mathrm{T}$ cells in lesional skin, the resulting gene expression data could not be informative.

An alternative to skin biopsy analysis has been the analysis of gene expression profiles of peripheral blood mononuclear cells (PBMCs) from patients and controls [22-25]. This approach is of particularly interest in immune-mediated diseases like psoriasis, since it is a less invasive approach and, consequently, predictive profiles can have a better translation into the clinical setting. However, most $\mathrm{T}$ cells circulating in the peripheral blood are in a quiescent state [26], with scarce gene expression activity [27]. Consequently, relevant pathogenic processes like $\mathrm{T}$ cell activation might not well be studied through this approach.

In the present study we have used in vitro activated $\mathrm{T}$ cells from patients and healthy controls to characterize the gene expression profile associated with psoriasis. Using an independent cohort of patients and controls we have validated the most significant genes associated with this differential regulatory activity. Finally, using different genomic approaches we have characterized the gene expression networks and biological pathways that characterize this key aspect of psoriasis biology.

\section{Results}

Gene expression profiles from in vitro activated $\mathrm{T}$ cells were obtained from 17 psoriasis patients and 7 healthy controls using Illumina HT-12 v4 microarrays. From each individual gender, age was collected, and Psoriasis Area and Severity Index (PASI) score was determined for each patient at the time of sample collection (Additional file 1). There were no statistically significant differences $(P>0.05)$ in age or gender between the microarray and validation case control samples. Also, the PASI score was not significantly different between discovery and validation patient samples. From each RNA sample, a total of 47,321 transcripts were quantified and 23,554 probes were called as expressed in the sample (i.e. present in 2 or more individuals) according to the Illumina expression score and selected for statistical analysis.

\section{Differentially expressed genes in in vitro activated T cells}

A total of 42 genes were found to be significantly associated with psoriasis (FDR p-value $<0.1$, absolute fold change $>1.2$, Additional file 2). From these, 25 were found to be up-regulated in $\mathrm{T}$ cells from psoriasis patients and 17 genes were down-regulated in patients compared to controls. The genes with FDR p-value $<$ 0.05 showing the largest change in gene expression (i.e. absolute fold change $>1.2$ ) are shown in Table 1 .

\section{Validation of genes associated with psoriasis in T cells}

From the list of significant differentially expressed genes, we selected those genes showing a high level of statistical association (Pcorrected $<0.005$ ) with the largest absolute change in expression (i.e. Fold Change $(\mathrm{FC})>1.3$ ) for validation. These genes were spermatogenesis associated serine-rich 2-like (SPATS2L, FC $=1.37$, Pcorrected $=$ $0.0009)$, Kruppel-like factor $6(K L F 6, \mathrm{FC}=1.32$, $\mathrm{p}$-value $=$ $0.0012), S P 140$ nuclear body protein $(S P 140, \mathrm{FC}=1.38$, $\mathrm{p}$-value $=0.0025)$ and RAR-related orphan receptor A $($ RORA, FC $=1.31, \mathrm{p}$-value $=0.0041) \quad($ Figure 1a) genes. Using the same set of samples from the microarray analysis we first performed a RT-PCR technical validation study. We were able to validate the differential expression observed for SPATS2L $(\mathrm{FC}=1.39, \quad \mathrm{p}$-value $=$ 0.0006), KLF6 $(\mathrm{FC}=1.24, \mathrm{p}$-value $=0.022)$ and $S P 140$ $(\mathrm{FC}=1.34, \mathrm{p}$-value $=0.019)$ genes (Figure $1 \mathrm{~b}) . R O R A$ differential expression was not validated ( $\mathrm{p}$-value $>0.05$ ) and, consequently, we did not perform biological validation on this sample.

After technical validation, we used an independent sample of patients and controls recruited from the same University Hospitals to validate the association of SPATS2L, KLF6 and SP140 genes with the activation of $\mathrm{T}$ cells in psoriasis. Using in vitro activated $\mathrm{T}$ cells from 8 patients and 8 controls we significantly validated the overexpression of SPATS2L $(\mathrm{FC}=1.58, \mathrm{p}$-value $=0.0014)$ and KLF6 $(\mathrm{FC}=1.23$, $\mathrm{p}$-value $=0.024)$ genes in psoriasis (Figure 1b). SP140 did not show significant differential expression in the independent replication cohort.

\section{Identification of SPAT2SL and KLF6 coexpression networks}

Since SPAT2SL and KLF6 have not been previously associated to the $\mathrm{T}$ lymphocyte activation process, we aimed 
Table 1 List of most significantly up-regulated genes in in vitro activated T cells of psoriasis patients compared to healthy controls

\begin{tabular}{|c|c|c|c|c|}
\hline Probe & Gene & Fold change & P value* & Full name \\
\hline \multicolumn{5}{|c|}{ Upregulated genes } \\
\hline ILMN_1683678 & SPATS2L & 1.37 & 0.0009 & Spermatogenesis associated, serine-rich 2-like \\
\hline ILMN_1735014 & KLF6 & 1.32 & 0.0012 & Kruppel-like factor 6 \\
\hline ILMN_1703263 & SP140 & 1.38 & 0.0025 & SP140 nuclear body protein \\
\hline ILMN_2322498 & RORA & 1.31 & 0.0041 & RAR-related orphan receptor A \\
\hline ILMN_2246956 & $B C L 2$ & 1.23 & 0.0062 & B-cell CLL/lymphoma 2 \\
\hline ILMN_2397721 & GLB1 & 1.23 & 0.0062 & Galactosidase, beta 1 \\
\hline ILMN_1781285 & DUSP1 & 1.21 & 0.0071 & Dual specificity phosphatase 1 \\
\hline ILMN_2321064 & $B A X$ & 1.24 & 0.0096 & BCL2-associated $X$ protein \\
\hline ILMN_1731107 & CCDC92 & 1.33 & 0.0136 & Coiled-coil domain containing 92 \\
\hline ILMN_1729749 & HERC5 & 1.72 & 0.0267 & Hect domain and RLD 5 \\
\hline ILMN_2095660 & TMEM156 & 1.29 & 0.0453 & Transmembrane protein 156 \\
\hline ILMN_1681301 & AIM2 & 1.42 & 0.0464 & Absent in melanoma 2 \\
\hline ILMN_1767470 & SCPEP1 & 1.26 & 0.0469 & Serine carboxypeptidase 1 \\
\hline \multicolumn{5}{|c|}{ Downregulated genes } \\
\hline ILMN_1778617 & TAF9 & -1.25 & 0.0009 & $\begin{array}{l}\text { TAF9 RNA polymerase II, TATA box binding protein } \\
\text { (TBP)-associated factor, 32kDa }\end{array}$ \\
\hline ILMN_2143250 & FAR1 & -1.20 & 0.0207 & Fatty acyl CoA reductase 1 \\
\hline ILMN_1720114 & GMNN & -1.20 & 0.0269 & Geminin \\
\hline ILMN_2135175 & SNORD36A & -1.27 & 0.0303 & Small nucleolar RNA, C/D box 36A \\
\hline
\end{tabular}

*Multiple-test corrected p-value.

List of genes with multiple-test corrected $p$-value $<0.05$ and absolute change $>1.2$. Genes are classified into up-regulated or down-regulated groups and sorted by their statistical significance. Genes that were selected to validate are highlighted in bold.

to identify and analyze the gene expression network associated to each of the two genes. For this objective we used the weighted correlation analysis implemented in the WGCNA method [28]. After filtering for the most informative genes (i.e. coefficient of variation $>0.1, \mathrm{n}=$ 4,512 genes), we identified a total of 20 different gene expression modules (Additional file 3). Using the eigenvectors derived from each gene expression module we evaluated the association to different clinical and epidemiological variables (Additional file 4). From these, we found four modules significantly associated with the presence of psoriasis ( $\mathrm{p}$-value $<0.05$, Additional files 5 and 6). These four modules were not associated with any of the other tested covariates. The first of these modules $(\mathrm{M} 1, \mathrm{p}$-value $=0.00051)$ included 54 genes and was the most enriched in genes differentially expressed between psoriasis cases and controls $(59.3 \%$ at nominal $\mathrm{p}$-value $<0.05)$. The second module $(\mathrm{M} 2, \mathrm{p}$-value $=$ 0.0038 ) consisted in 92 genes from which $37 \%$ were differentially expressed. The third module $(\mathrm{M} 3, \mathrm{p}$-value $=$ 0.011 ) included 31 genes and $29 \%$ from them showed differential expression. Finally, the fourth module (M4, $\mathrm{p}$-value $=0.047)$ included a total of 115 genes, but with a lower percentage showing differential expression between cases and controls (24.3\%).
SPATS2L was found to belong to M1 gene expression module, while KLF6 was found to be coexpressed with genes in module M2. The fourth module associated with psoriasis, M4, included the genome-wide significant RORA gene that could not be technically validated by RTPCR.

\section{Gene Ontology analyses of SPATS2L and KLF6 coexpression networks}

In order to functionally characterize SPATS2L and KLF6 coexpression networks we performed Gene Ontology (GO) enrichment analysis using DAVID functional annotation tool [29]. Table 2 shows the most significantly enriched GO terms related to biological processes. The complete list, with the significant GO terms associated to cellular component and molecular function, is provided in the Additional file 7.

In the SPATS2L coexpression network, eight GO terms were found to be significantly enriched ( $\mathrm{p}$-values from 0.01 to 0.05 ). Most of these GO terms were related to different aspects of chromosome and ribosomal biology (e.g., GO:0051276, chromosome organization, $\mathrm{p}$-value $=0.019$; GO:0042273, ribosomal biogenesis, $\mathrm{p}$-value $=0.021$ ).

In the KLF6 coexpression network $35 \mathrm{GO}$ terms were found to be significantly enriched ( $\mathrm{p}$-values ranging from $3.95 \times 10 \mathrm{e}-6$ to 0.05$)$. In this network, the most significantly 

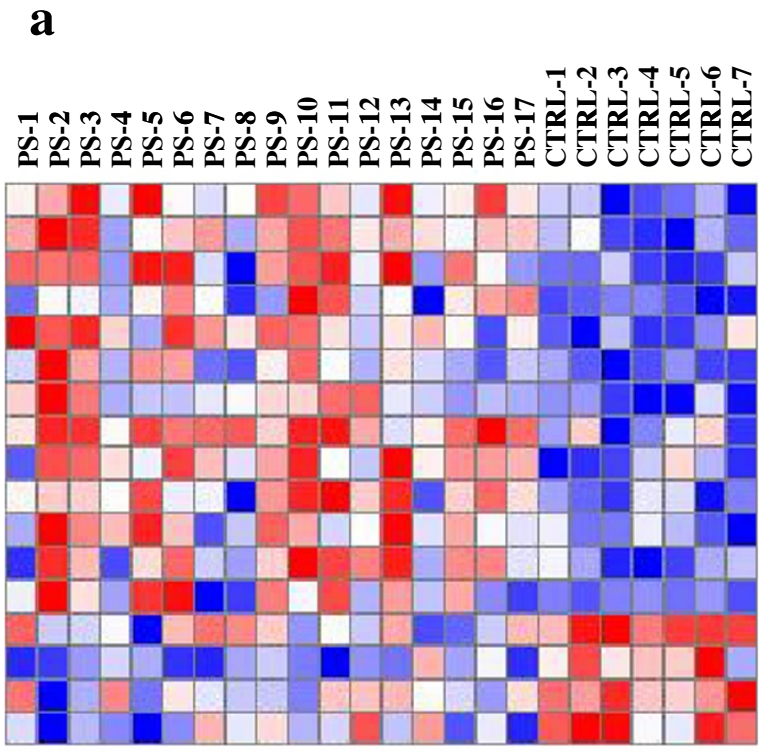

$\begin{array}{lll}\text { Symbol } & \text { FC } & \text { p-value } \\ \text { SPATS } 2 L & 1.37 & 0.0009 \\ \text { KLF6 } & 1.32 & 0.0012 \\ \text { SP140 } & 1.38 & 0.0025 \\ \text { RORA } & 1.31 & 0.0041 \\ \text { BCL2 } & 1.23 & 0.0062 \\ \text { GLB1 } & 1.23 & 0.0062 \\ \text { DUSP1 } & 1.21 & 0.0071 \\ \text { BAX } & 1.24 & 0.0096 \\ \text { CCDC92 } & 1.33 & 0.0136 \\ \text { HERC5 } & 1.72 & 0.0267 \\ \text { TMEM156 } & 1.29 & 0.0453 \\ \text { AIM2 } & 1.42 & 0.0464 \\ \text { SCPEP1 } & 1.26 & 0.0469 \\ \text { TAF9 } & -1.25 & 0.0009 \\ \text { FARI } & -1.20 & 0.0207 \\ \text { GMNN } & -1.20 & 0.0269 \\ \text { SNORD36A } & -1.27 & 0.0303\end{array}$
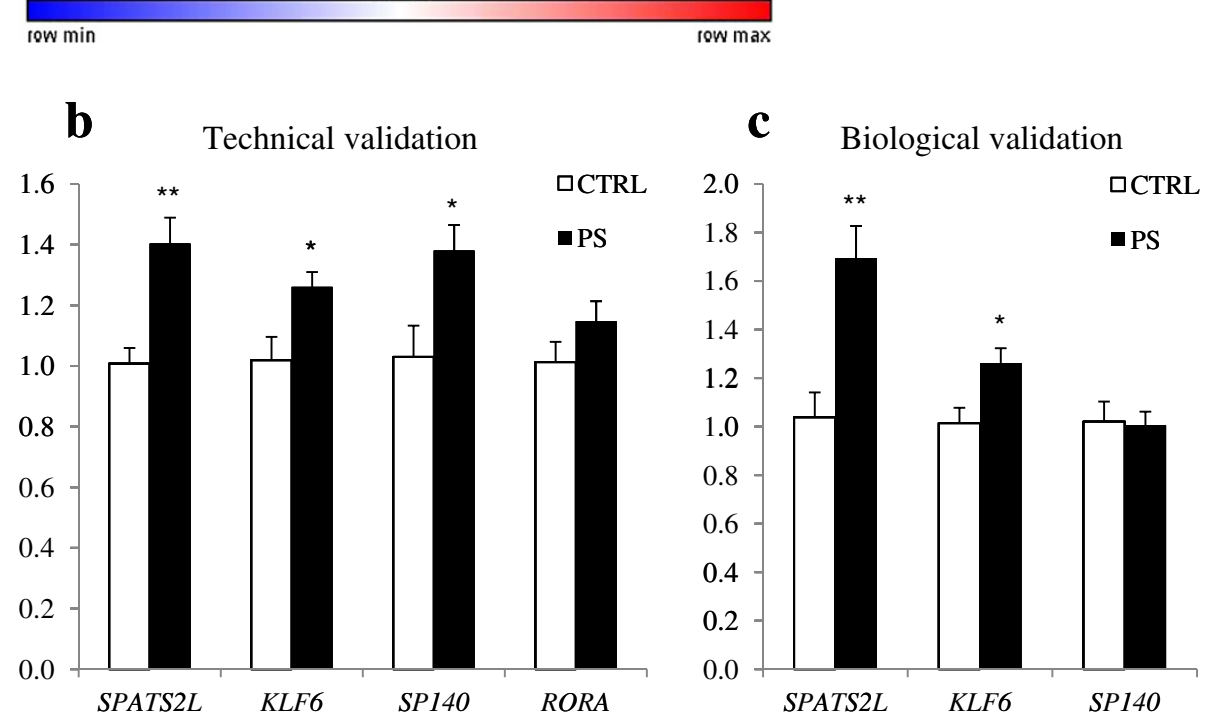

Figure 1 Validation of the most significantly up-regulated genes in in vitro activated T cells of psoriasis patients compared to healthy controls. (a) Heat map of microarray expression data for the genes most significantly associated with psoriasis (FDR p-value $<00.5, F C H>1.2$ ). The gene expression values for each gene have been scaled using the minimum and maximum values in order to represent a normalized gene expression gradient (b) Technical validation of SPATS2L, KLF6, SP140 and RORA genes by RT-PCR (Psoriasis $\mathrm{n}=17$, and Controls $\mathrm{n}=7$ ). (c) Biological validation of SPATS2L, KLF6 and SP140 genes by RT-PCR in an independent cohort of psoriasis patients and healthy controls $(n=8$ each). ${ }^{* *} p<0.01$ and ${ }^{*} p<0.05$.

enriched GO terms ( $\mathrm{p}$-value $<0.01$ ) were involved in lipid synthesis ( $n=7$ ontologies), followed by GO terms associated with amino acid biosynthesis ( $\mathrm{n}=6$ ontologies), hydrogen sulphide process ( $\mathrm{n}=2$ ontologies) and endoplasmic reticulum stress $(\mathrm{n}=1$ ontology).

Gene set enrichment analysis of the T cell transcriptome In order to further investigate the biological basis of the gene expression differences observed between $\mathrm{T}$ cells from psoriasis patients and controls, we analyzed the global transcriptome using the GSEA approach [30,31].
A total of 55 gene sets were found to be significantly enriched between cases and controls (False Discovery Rate, FDR < 0.05) (Table 3). From these, four gene sets were significant at a FDR $<0.0001$ all belonging to Reactome database [32] pathway definitions: Cytokine Signaling in Immune System, Interferon Signaling, Interferon Gamma Signaling and Interferon Alpha/Beta Signaling.

The top gene sets identified using GSEA encoded for cytokine signalling-related genes, more specifically, for genes associated with alpha/beta and gamma interferon signalling. Consistent with this strong interferon response, 
Table 2 Summary of the enriched GO Biological Process terms in SPATS2L and KLF6 coexpression networks

\begin{tabular}{|c|c|c|c|}
\hline \multicolumn{4}{|l|}{ Biological process in SPATS2L network } \\
\hline Term & Count & $\%$ & p-value \\
\hline GO:0051276 chromosome organization & 5 & 12.8 & $1.90 \mathrm{E}-02$ \\
\hline GO:0042273 ribosomal large subunit biogenesis & 2 & 5.1 & $2.12 \mathrm{E}-02$ \\
\hline GO:0006461 protein complex assembly & 5 & 12.8 & 2.17E-02 \\
\hline GO:0070271 protein complex biogenesis & 5 & 12.8 & 2.17E-02 \\
\hline GO:0042254 ribosome biogenesis & 3 & 7.7 & 2.79E-02 \\
\hline GO:0034622 cellular macromolecular complex assembly & 4 & 10.3 & 2.99E-02 \\
\hline GO:0034621 cellular macromolecular complex subunit organization & 4 & 10.3 & 4.01E-02 \\
\hline GO:0006325 chromatin organization & 4 & 10.3 & 4.63E-02 \\
\hline \multicolumn{4}{|l|}{ Biological process in KLF6 network } \\
\hline Term & Count & $\%$ & p-value \\
\hline GO:0006695 cholesterol biosynthetic process & 5 & 6.33 & $3.95 \mathrm{E}-06$ \\
\hline GO:0016126 sterol biosynthetic process & 5 & 6.33 & $1.35 \mathrm{E}-05$ \\
\hline GO:0044271 nitrogen compound biosynthetic process & 8 & 10.13 & 4.08E-04 \\
\hline GO:0006694 steroid biosynthetic process & 5 & 6.33 & 4.45E-04 \\
\hline GO:0008203 cholesterol metabolic process & 5 & 6.33 & $6.01 \mathrm{E}-04$ \\
\hline GO:0016125 sterol metabolic process & 5 & 6.33 & 8.54E-04 \\
\hline GO:0009070 serine family amino acid biosynthetic process & 3 & 3.80 & 9.36E-04 \\
\hline GO:0008652 cellular amino acid biosynthetic process & 4 & 5.06 & $1.28 \mathrm{E}-03$ \\
\hline GO:0008610 lipid biosynthetic process & 7 & 8.86 & $2.30 \mathrm{E}-03$ \\
\hline GO:0008299 isoprenoid biosynthetic process & 3 & 3.80 & $3.16 \mathrm{E}-03$ \\
\hline GO:0009309 amine biosynthetic process & 4 & 5.06 & 4.80E-03 \\
\hline GO:0006094 gluconeogenesis & 3 & 3.80 & $4.92 \mathrm{E}-03$ \\
\hline GO:0009069 serine family amino acid metabolic process & 3 & 3.80 & $5.31 \mathrm{E}-03$ \\
\hline GO:0019319 hexose biosynthetic process & 3 & 3.80 & $7.50 \mathrm{E}-03$ \\
\hline GO:0070814 hydrogen sulfide biosynthetic process & 2 & 2.53 & $8.41 \mathrm{E}-03$ \\
\hline GO:0070813 hydrogen sulfide metabolic process & 2 & 2.53 & $8.41 \mathrm{E}-03$ \\
\hline GO:0019344 cysteine biosynthetic process & 2 & 2.53 & $8.41 \mathrm{E}-03$ \\
\hline GO:0034976 response to endoplasmic reticulum stress & 3 & 3.80 & 8.97E-03 \\
\hline
\end{tabular}

List of enriched GO terms related to biological process (BP) in the SPATS2L ( $p$-value $<0.05$ ) and KLF6 coexpression networks ( $p$-value $<0.01)$. For each term, the number of genes present in the network (count), the percentage of enrichment (\%) and the associated p-value are indicated.

a group of gene sets related to innate immune system $($ FDR $=0.0014)$ and RIG and NOD-like receptor pathways (FDR $=0.0005$ and 0.0058 , respectively) were also among the most significantly enriched.

$\mathrm{T}$ cell activation in psoriasis patients also resulted in a significant enrichment of genes involved in PPAR $\alpha$ mediated activation of gene expression $(F D R=0.0043)$ and cholesterol biosynthesis (FDR $=0.0063)$. Cholesterol biosynthesis was also the most significant GO biological process associated with KLF6 coexpression network. Finally, protein misfolding in the endoplasmic reticulm was also a common pathway detected by GSEA (i.e. unfolded protein response, $F D R=0.012$ ) and in the $G O$ analysis of KLF6 network.

\section{Discussion}

It is widely accepted that psoriasis is a T-cell mediated disease, in which lymphocytes continuously infiltrate the skin promoting and perpetuating inflammation. However, little is known yet on the gene expression regulation that characterizes $\mathrm{T}$ cell activation in psoriasis. Most of the whole genome gene expression studies in psoriasis performed to date have been principally been conducted in skin, where the $\mathrm{T}$ cell-associated gene expression is obscured by other more predominant cell types like hyperproliferative keratinocytes. Alternatively, in gene expression studies using freshly isolated blood lymphocytes, most $\mathrm{T}$ cells are found in a quiescent state and might not reflect gene expression profiles relevant 
Table 3 Summary of the GSEA-identified gene sets in in vitro activated T cells of psoriasis patients compared to healthy controls

\begin{tabular}{|c|c|c|c|}
\hline Name & NES & NOM p-val & FDR q-val \\
\hline \multicolumn{4}{|l|}{ General processes of immune system } \\
\hline Reactome cytokine signaling in immune system & 2.498 & 0.000 & 0.0000 \\
\hline KEGG cytokine cytokine receptor interaction & 1.980 & 0.000 & 0.0130 \\
\hline KEGG hematopoietic cell lineage & 1.917 & 0.000 & 0.0167 \\
\hline KEGG cell adhesion molecules cams & 1.903 & 0.000 & 0.0173 \\
\hline \multicolumn{4}{|l|}{ Infectious and autoimmune diseases } \\
\hline KEGG autoimmune thyroid disease & 1.996 & 0.002 & 0.0126 \\
\hline KEGG allograft rejection & 1.866 & 0.000 & 0.0234 \\
\hline KEGG leishmania infection & 1.961 & 0.000 & 0.0121 \\
\hline KEGG viral myocarditis & 1.900 & 0.000 & 0.0175 \\
\hline \multicolumn{4}{|l|}{$\mathrm{T}$ cell activation } \\
\hline Reactome downstream TCR signaling & 1.869 & 0.000 & 0.0232 \\
\hline PID NFAT TF pathway & 1.903 & 0.000 & 0.0179 \\
\hline PID AP1 pathway & 1.936 & 0.000 & 0.0148 \\
\hline Biocarta CTLA4 pathway & 1.854 & 0.000 & 0.0253 \\
\hline \multicolumn{4}{|l|}{ Innate immune system activation } \\
\hline Reactome innate immune system & 2.145 & 0.000 & 0.0014 \\
\hline Reactome inflammasomes & 1.905 & 0.000 & 0.0180 \\
\hline Reactome antiviral mechanism by IFN stimulated genes & 1.915 & 0.000 & 0.0160 \\
\hline Reactome RIG I MDA5 mediated induction of IFN alpha beta pathways & 2.212 & 0.000 & 0.0005 \\
\hline KEGG RIG I like receptor signaling pathway & 2.144 & 0.000 & 0.0012 \\
\hline Reactome negative regulators of RIG I MDA5 signaling & 2.123 & 0.000 & 0.0018 \\
\hline KEGG NOD like receptor signaling pathway & 1.953 & 0.000 & 0.0128 \\
\hline Reactome nucleotide binding domain leucine rich repeat containing receptor NLR signaling pathways & 2.048 & 0.000 & 0.0058 \\
\hline KEGG toll like receptor signaling pathway & 1.802 & 0.000 & 0.0398 \\
\hline Reactome TRAF6 mediated IRF7 activation & 1.875 & 0.002 & 0.0226 \\
\hline KEGG cytosolic DNA sensing pathway & 1.781 & 0.002 & 0.0449 \\
\hline \multicolumn{4}{|l|}{ Cytokine signaling } \\
\hline KEGG JAK STAT signaling pathway & 1.977 & 0.000 & 0.0119 \\
\hline PID IL12 STAT4 pathway & 1.775 & 0.004 & 0.0454 \\
\hline Reactome interferon signaling & 2.701 & 0.000 & 0.0000 \\
\hline Reactome interferon alpha beta signaling & 2.760 & 0.000 & 0.0000 \\
\hline Reactome interferon gamma signaling & 2.303 & 0.000 & 0.0000 \\
\hline PID IFNG pathway & 1.787 & 0.002 & 0.0434 \\
\hline PID SMAD2 3 nuclear pathway & 2.094 & 0.000 & 0.0031 \\
\hline Reactome transcriptional activity OF SMAD2 SMAD3 SMAD4 heterotrimer & 1.794 & 0.002 & 0.0421 \\
\hline Reactome SMAD2 SMAD3 SMAD4 heterotrimer regulates transcription & 1.771 & 0.012 & 0.0451 \\
\hline \multicolumn{4}{|l|}{ Differentiation, proliferation and apoptosis } \\
\hline PID notch pathway & 1.916 & 0.000 & 0.0163 \\
\hline PID Betacatenin Nuc pathway & 1.844 & 0.002 & 0.0271 \\
\hline Biocarta gleevec pathway & 1.862 & 0.000 & 0.0237 \\
\hline PID CMYB pathway & 1.842 & 0.000 & 0.0271 \\
\hline Reactome YAP1 AND WWTR1 TAZ stimulated gene expression & 1.804 & 0.008 & 0.0400 \\
\hline PID P53 downstream pathway & 1.772 & 0.000 & 0.0452 \\
\hline
\end{tabular}




\begin{tabular}{|c|c|c|c|}
\hline \multicolumn{4}{|l|}{ Metabolism } \\
\hline Reactome cholesterol biosynthesis & 2.050 & 0.000 & 0.0063 \\
\hline Reactome fatty acid triacylglycerol and ketone body metabolism & 1.961 & 0.000 & 0.0128 \\
\hline Reactome metabolism of lipids and lipoproteins & 1.927 & 0.000 & 0.0157 \\
\hline KEGG biosynthesis of unsaturated fatty acids & 1.790 & 0.006 & 0.0429 \\
\hline KEGG glycine serine and threonine metabolism & 1.877 & 0.000 & 0.0227 \\
\hline KEGG metabolism of xenobiotics by cytochrome P450 & 1.924 & 0.002 & 0.0157 \\
\hline Reactome PPARA activates gene expression & 2.076 & 0.000 & 0.0043 \\
\hline Biocarta PPARA pathway & 1.780 & 0.006 & 0.0444 \\
\hline \multicolumn{4}{|l|}{ Unfolded protein response } \\
\hline Reactome unfolded protein response & 1.972 & 0.000 & 0.0120 \\
\hline Reactome perk regulated gene expression & 1.872 & 0.000 & 0.0230 \\
\hline Reactome activation of genes by ATF4 & 1.896 & 0.000 & 0.0180 \\
\hline PID ATF2 pathway & 1.977 & 0.000 & 0.0125 \\
\hline \multicolumn{4}{|l|}{ Other } \\
\hline KEGG intestinal immune network for IGA production & 1.996 & 0.000 & 0.0117 \\
\hline KEGG Iysosome & 1.951 & 0.000 & 0.0127 \\
\hline Reactome generic transcription pathway & 1.813 & 0.000 & 0.0372 \\
\hline PID HIF1 TFPATHWAY & 1.778 & 0.002 & 0.0444 \\
\hline Reactome RORA activates circadian expression & 1.773 & 0.007 & 0.0457 \\
\hline
\end{tabular}

List of the enriched gene sets in psoriasis phenotype compared to control phenotype showing a FDR $<0.05$. Gene sets are classified according to the processes where they are involved. Normalized enrichment score (NES), nominal p-value (NOM p-value) and false discovery rate (FDR q-value) are indicated.

to the disease. In the present study, we aimed to overcome these limitations by characterizing the differential gene expression profile of in vitro activated $\mathrm{T}$ cells in psoriasis patients and controls. To our knowledge, it is the first study to analyze $\mathrm{T}$ cell activation in psoriasis using whole-genome gene expression analysis.

In the microarray analysis of in vitro activated $\mathrm{T}$ cells we were able to identify 42 genes differentially expressed between psoriasis patients and controls. Using an independent sample of patients and controls we validated the association of the two genes most highly overexpressed in psoriasis T cells, SPATS $2 L$ and KLF6. It is the first time that SPATS $2 L$ and KLF6 genes have been associated with the $\mathrm{T}$ cell activation process in psoriasis.

To date, little is known on the biological functionality of SPATS2L. In our study, combining gene coexpression network analysis and Gene Ontology enrichment we found that SPATS2L gene network is associated to processes associated with the activation of gene expression including chromosomal organization and ribosomal biogenesis. This potential association of SPATS2L with ribosomal processes and translational control has been previously described in gene-trap experiments conducted in myoblasts under oxidative stress [33]. Ribosomal biogenesis has been proved to be critical in the control of cell growth and cell proliferation [34], and in
T cells, is one of the most significantly up-regulated cellular processes during cell-cycle entry [35]. Therefore, the overexpression of SPATS $2 L$ in activated T cells from psoriasis patients could be contributing to an earlier activation of these lymphocytes compared to healthy controls.

Consistently with this potential role in altered cell activation, there is evidence that SPATS2L is associated with lymphoproliferative-type of disorders. Analyzing the overexpression of SPATS $2 L$ in different human diseases, we found that SPATS $2 L$ is one of the genes most significantly overexpressed in lymphocytes from patients with chronic lymphocytic leukemia (CLL) compared to cells from normal controls (Additional file 8) [36]. Also, SPATS2L is found to be significantly overexpressed in a mouse model of T-cell acute lymphoblastic leukemia (Additional file 8) [37]. Furthermore, gene expression data from leukemia cell lines treated with anti-leukemic agent Imatinib, shows a significant downregulation of SPATS2L (Additional file 8). Finally, a recent genomewide association study (GWAS) data on risk loci for lymphoblastic leukemia has shown a significant association of a Single Nucleotide Polymorphism in the SPATS2L locus [38]. Together, these results show that $S P A T S 2 L$ functionality is strongly associated with the aberrant activation of lymphocytes. 
Interestingly, there is evidence that psoriasis and lymphoproliferative disorders could share common genetic basis. Patients with psoriasis have an increased risk of developing leukemia, in particular lymphoma [39]. In a GWAS performed in follicular lymphoma, the region with most significant association was located near the psoriasis susceptibility region 1 (PSORS1) in chromosome 6 [40]. Furthermore, a fairly common manifestation of certain leukemias, including lymphocytic leukemias, is the infiltration of neoplastic leukocytes in skin tissue, also known as leukemia cutis [41]. Therefore, it is tempting to speculate that SPATS $2 L$ is involved in a common genetic pathway associated with psoriasis and lymphoproliferative-type of disorders. This pathway would therefore participate in the aberrant lymphocyte activity observed in both diseases and the preferential infiltration of skin tissue by these altered lymphocytes.

KLF6 encodes a member of Kruppel family of transcription factors [42], several of which have been previously implicated in T lymphocyte biology [43]. KLF6 has been previously shown to be expressed in lymphoid cells $[44,45]$ and, similarly to SPATS $2 L$, there is also evidence of differential expression of KLF6 in T cells from CLL patients compared to controls [46]. Compared to SPATS2L however, KLF6 coexpression network showed a highly significant Gene Ontology enrichment, mainly in ontologies related to the biosynthesis of lipids and proteins. These biosynthetic processes are induced in cells that are stimulated to rapidly grow and proliferate, as it is the case of activated T cells [47-50]. Two other significant biological processes enriched in the KLF6 network were endoplasmic reticulum stress and biosynthesis of hydrogen sulfide. These processes are linked to another process also found to be enriched by psoriasis in GSEA analysis which is the unfolded protein response (UPR) [51,52]. Interestingly, the expression of KLF6 and other KLF members has been previously reported to be up-regulated when UPR is induced by different drugs, such as tunicamycin [53] or thapsigargin (Additional file 8) [54]. On the basis of this information, it is possible that in $\mathrm{T}$ cells from psoriasis patients, KLF6 up-regulation may also be induced by an UPR, in this case, triggered by the increase in protein-folding load required upon $\mathrm{T}$ cell activation.

We have investigated the biological processes that take place during $\mathrm{T}$ cell activation associated with psoriasis using both an unsupervised approach which does not use sample information, WGCNA, as well as a supervised approach, GSEA, which compares gene sets overrepresented in cases compared to controls. Even though these are clearly different functional analysis, they resulted in the identification of common biological processes. One of these common biological processes was the biosynthesis of lipids and proteins. For example, cholesterol biosynthesis was the most significantly enriched process in KLF6 coexpression network and was also detected by GSEA as one of the most enriched processes in T cells from psoriasis patients. This enrichment in genes involved in biosynthetic processes could be attributed to an early activation of $\mathrm{T}$ cells from psoriasis patients. Another common biological pathway identified in both approaches was the endoplasmic reticulum stress and unfolded protein response. Interestingly, these two processes, which may result from the increased protein-folding load required in activated $\mathrm{T}$ cells, have been associated with a number of chronic inflammatory and autoimmune diseases like spondyloarthritis, rheumatoid arthritis, inflammatory bowel diseases or multiple sclerosis [55-57].

The most significantly enriched gene sets identified by GSEA were, however, related to cytokine signalling $(\mathrm{FDR}<0.0001)$, more specifically, to genes involved in the interferon signalling pathway (IFN pathway FDR < 0.0001 ; IFNG signalling, FDR $<0.0001$; IFNA/B signalling, FDR $<0.0001)$. This result is fully consistent with the strong interferon-signature detected in psoriasis skin lesions [20]. In line with this observation, GSEA results also show evidence of an association with IL12-STAT4 pathway, an interferon gamma inducing pathway, although at much lower significance. IFN has been shown to drive activation and differentiation of IL17 and IL22 producing $\mathrm{T}$ cells [58], and to increase keratinocyte responsiveness to IL22 which leads to the observed hyperproliferation [59]. In line with these observations, GSEA analysis also identified an enrichment of innate immune sensing mechanisms related to the production of IFN like RIG-I receptor signaling pathway $(F D R=0.0005)$ [60] and the NLR family of receptors like NOD2 [61] $(F D R=0.0058)$. Together, these results suggest that IFN response pathway is more strongly activated in $\mathrm{T}$ cells from psoriasis patients than normal healthy controls.

The four $\mathrm{T}$ cell gene networks associated with psoriasis risk identified through WGCNA analysis were found to be highly enriched for genes associated to the response to virus biological process (Additional file 9). A total of 14 genes (BCL2, ISG15, EIF2AK2, IFIH1, IRF7, ISG20, IFI35, IFI44, MX1, MX2, RSAD2, STAT1, STAT2 and TRIM5) out of 61 overexpressed by $\mathrm{T}$ cells $(\mathrm{P}<1 \mathrm{e}-$ 16 , test for enrichment) were associated to the response to a viral infection. This result is in accordance with the recent evidence implicating an increased antiviral activity in psoriatic skin compared to skin from normal controls or patients with atopic dermatitits [62]. In this model, keratinocytes are rendered hyperresponsive towards an antiviral response after being conditioned from T helper 17 (Th17) cell-type cytokines.

In the last years the two newly characterized T-cell subsets, Th17 and Th22, have been increasingly 
implicated in the pathogenesis of psoriasis. While our approach does not provide direct evidence of an increase of either cell subset, it describes an increased activation of precursor cells in affected individuals. It is therefore likely that this differential activity predisposes to these two particular cell types once in the skin microenvironment of psoriasis patients. Additional studies will be needed to confirm this hypothesis. Another limitation of our study is that we analyzed the global expression changes of all activated $T$ cells, regardless of the specific subphenotype. While we found no statistically significant differences in CD4+ or CD8+ percentages in cases compared to controls (data not shown), it is possible that specific subphenotypes are differently represented between the two groups. Therefore, future studies will be performed to identify which cell subtype/s are responsible for each of the differentially expressed genes and genetic pathways associated with psoriasis in our study.

\section{Conclusions}

In the present study, we have investigated for the first time the gene expression profile specifically associated with $\mathrm{T}$ cell activation in psoriasis. We have identified SPATS2L and KLF6 as the two genes most significantly overexpressed in activated $\mathrm{T}$ cells from psoriasis patients and we have validated this overexpression in an independent case-control cohort. Functional analysis of the gene coexpression networks, suggest that SPATS2L and KLF6 might be involved in an early activation process. Global functional enrichment also shows a strong prevalence of interferon cytokine mediated signalling in activated $\mathrm{T}$ cells from psoriasis compared to controls. The results of this study associate new biological pathways with psoriasis etiology and establish new lines of research that will clearly lead to an improved knowledge of the biological basis of this disease and, hopefully, to improved therapies in the near future.

\section{Methods}

\section{Subjects}

Fifteen healthy controls and twenty-five psoriasis patients were included in the present study. Controls (mean \pm SD age $40.8 \pm 8.8$ years; 9 men and 6 women) didn't have psoriasis or any other immune mediated inflammatory disease and were collected from medical staff at the Vall d'Hebron Hospital (Barcelona, Spain). Psoriasis patients (mean \pm SD age $46 \pm 8.4$ years; 14 men and 11 women), were recruited from the outpatient's clinics of the dermatology departments from Santa Creu i Sant Pau Hospital (Barcelona, Spain) and Germans Trias i Pujol Hospital (Badalona, Spain). All selected patients had chronic plaque psoriasis as diagnosed by a dermatologist, affecting torso and/or extremities with at least one year of duration. Patients with a single clinical localization of plaque psoriasis (i.e. scalp, face, palmoplantar), with exclusively inverse plaque psoriasis or with an inflammatory bowel disease were excluded. All patients were $>18$ years old at the time of recruitment, although disease onset could have occurred at an earlier age.

Informed consent was obtained from all participants and protocols were reviewed and approved by the Vall d'Hebron University Hospital ethics committee. The present study was conducted according to the Declaration of Helsinki principles. Additional file 1 summarizes the main clinical features of patients and controls (age, gender, PASI score).

\section{Cell culture}

Peripheral blood mononuclear cells (PBMCs) were isolated from whole blood of patients and controls by centrifugation on a Ficoll-Paque PLUS (GE Healthcare Biosciences $\mathrm{AB}$, Uppsala, Sweden) density gradient. PBMCs were then seeded in complete RPMI 1640 medium supplemented with $100 \mathrm{U} / \mathrm{ml}$ penicillin, $100 \mathrm{U} / \mathrm{ml}$ streptomycin, $10 \%$ fetal bovine serum, $2 \mathrm{mM}$ L-glutamine and $50 \mu \mathrm{M}$ mercaptoethanol, and activated with $1 \mu \mathrm{g} / \mathrm{ml}$ of anti-CD3 and anti-CD28 antibodies (eBioscience, San Diego, CA, USA). After 72 hours of stimulation, activated $\mathrm{T}$ cells were harvested by centrifugation and processed for flow cytometry study or frozen at $-20^{\circ} \mathrm{C}$ for subsequent RNA isolation.

\section{Flow cytometry analysis}

In order to characterize the $\mathrm{T}$ lymphocyte population present in cultured PBMCs samples and verify their activation after stimulation by $\mathrm{CD} 3$ and $\mathrm{CD} 28$ ligation, we performed flow cytometric analysis. In vitro activated cells were stained with anti-human CD4-PE (eBioscience, San Diego, CA, USA) and anti-human CD8-PerCP (Biolegend, San Diego, CA, USA) antibodies before acquisition (FC500 Flow Cytometer, Beckman Coulter, CA, USA) and analysis (Flowjo, Tree Star, Inc., Oregon, USA). Forward and side scatters were used to gate resting and blast lymphocytes, and $\mathrm{CD} 4+$ or CD8+ -type cells were counted within each gate. The average percentage of blasting cells was $75.94(+/-9.9)$ with $51.36 \%(+/-11.3)$ being $C D 4+$ and $37.87 \%(+/-10.7)$ corresponding to CD8+. No statistically significant differences were observed between cases and controls.

\section{RNA isolation}

Total RNA was isolated from previously activated $\mathrm{T}$ cells using the RNeasy mini kit (Qiagen, Hilden, Germany). RNA yield was assessed using a NanoDrop 1000 spectrophotometer (Thermo Fisher Scientific, USA) and RNA integrity was assessed using the RNA 6000 Nano LabChip Kit on an Agilent 2100 Bioanalyser (Agilent 
technologies, Waldbronn, Germany). All RNA samples had a RNA Integrity Number higher than 9 and were subsequently used for microarray analysis.

\section{Microarray analysis and differential gene expression determination}

Whole genome gene expression analysis of in vitro activated T cells for 17 psoriasis patients and 7 healthy controls was carried out by the HudsonAlpha Institute for Biotechnology using the Illumina HT-12 V4 microarrays (Illumina, USA). Briefly, the Illumina CustomPrep RNA amplification kit (Appiled Biosystems, USA) was used to generate biotinylated amplified complementary RNA (cRNA) from $200 \mathrm{ng}$ of the extracted total RNA. Labelled cRNA was then hybridized overnight to the Illumina microarrays, washed, blocked, stained and scanned using the Illumina BeadStation 500. The GenomeStudio software v2011.1 (Illumina, USA) was used to generate the raw data from the microarray scanned images. HT-12 V4 microarrays allow the simultaneous profiling of $>47,000$ transcripts, which include 28,688 well-established RefSeq annotations from the Human RefSeq (Rel 38). Sample labeling, microarray hybridization, scanning and raw data acquisition were performed at HudsohAlpha Institute for Biotechnology (Alabama, USA).

Data preprocessing, normalization and differential expression was performed using the R-statistical software v12.0 [63]. Log2-transformed data was normalized using the quantile normalization method [64]. Using the probe detection p-value calculated by GenomeStudio, only those transcripts present in 3 or more samples were considered for differential expression analysis. After filtering, a total of 25,539 transcripts were finally used to determine the associated gene expression profiles. Differential gene expression between cases and controls was tested using the $t$-test method implemented in the Bioconductor multtest package [65]. Correction for multiple testing was performed using the Benjamini and Hochberg False Discovery Rate method [66]. From these significant genes, only those having an absolute fold change $>1.2$ were considered to be differentially expressed between the two conditions.

\section{Real-time PCR validation}

200 ng of total RNA from 8 psoriasis cases and 8 controls were reverse-transcribed using the High Capacity cDNA Reverse Transcription Kit (Invitrogen, Carlsbad, CA) following the manufacturer's instructions. Real-time PCRs were performed on an ABI PRISM7900 Sequence Detection HT system (Applied Biosystems, Foster City, CA, USA) and using the TaqMan ${ }^{\circ}$ chemistry (Applied Biosystems, Foster City, CA, USA). The thermal cycle conditions were: $50^{\circ} \mathrm{C}$ for two minutes, $95^{\circ} \mathrm{C}$ for $10 \mathrm{mi}$ nutes and 40 cycles of $95^{\circ} \mathrm{C}$ for 15 seconds and $60^{\circ} \mathrm{C}$ for one minute. The selected TaqMan expression assays were: Hs01016366_m1 (SPATS2), Hs00296661_s1 (KLF6), Hs00916867_m1 (SP140), Hs00931153_m1 (RORA) and Hs01060665_g1 (ACTB). The method of 2 - [Delta] [Delta] Ct was used to obtain the mRNA expression of psoriasis samples relative to the control samples, normalizing the $\mathrm{Ct}$ values of target genes with those of the housekeeping gene actin beta $(A C T B)$.

\section{Gene expression network analysis}

Genes involved in a same biological process or genetic pathway tend to show correlated levels of expression. Using this property there are different methods that are able to build up networks of genes that better characterize the biological process of interest [67]. Such analysis can also provide a powerful approach for elucidating the unknown role of a given gene, based on the pathways or processes in which the other genes in the same network are involved. In the present study we have used the Weighted correlation network analysis (WGCNA) approach to infer the gene expression modules in activated $\mathrm{T}$ cells that are associated with psoriasis [28]. After calculating the co-expression matrix using Pearson's correlation method, a network adjacency is calculated by raising the co-expression similarity to a power $\beta$. Then using the scale-free topology criterion [68], the threshold parameter that defines the connectivity between genes is chosen. Once the threshold for connectivity is set, the coexpression network can be built. Within this network, clusters of densely connected genes (i.e. modules) are defined using unsupervised clustering and the Dynamic Tree Cut method [69]. Finally, for each gene expression module a summary measure or eigengene can be calculated using the first principal component of the corresponding expression matrix. Each eigengene was subsequently used to test for association with psoriasis and other relevant clinical and epidemiological covariates (age, gender, PASI score, \% of activated lymphocytes and \% CD4 or CD8 subsets). For binary variables the $t$-test was used, while for quantitative variables a linear regression approach was used.

In order to contextualize the gene networks associated with psoriasis, we performed a comprehensive search for previous studies having found evidence between the network genes and the disease. Using each gene annotation and the term 'psoriasis' we performed systematic queries using NCBI Pubmed database (October 2013). In order to reduce the presence of false positives only genes with absolute $\mathrm{FCH}>1.2$ were included. A total of 70 overexpressed genes and 22 underexpressed genes were queried.

\section{Functional annotation of expression modules}

Gene Ontology (GO) category enrichment analysis was performed using DAVID online tool (i.e. Database for 
Annotation, Visualization and Integrated Discovery, http://david.abcc.ncifcrf.gov/, Bethesda, MD, USA) [29]. Briefly, GO enrichment is calculated comparing the frequency of genes from each different GO present in the set of differently expressed genes to the frequency of genes for this same GO in the human genome background.

\section{Gene set enrichment analysis}

GSEA is a software method to analyze and interpret microarray data using biological knowledge [30,31]. Using the differential gene expression result between cases and controls and a list of predefined biological sets of genes, GSEA tests the overrepresentation each gene set in the group of most associated genes compared to the group of genes showing lower -or none- evidence of association. In the present study we used GSEA version 2.0.12 (www Broad Institute at MIT), with the $\mathrm{C} 2 \mathrm{cu}$ rated canonical pathway gene set (i.e. c2.cp.v3.1.symbols. gmt download), collapsing of dataset to gene symbols, weighted enrichment statistic and real gene list sorting. Maximum size of gene sets was set to 500 genes and minimum gene set size was set to 15 . Empirical significance values were calculated using 10,000 permutations.

\section{Availability of supporting data}

The datasets supporting the results of this article are available in NCBI's gene Expression Omnibus and are accessible through GEO Series accession number GSE47598 (http://www.ncbi.nlm.nih.gov/geo/query/acc. cgi?acc=GSE47598).

\section{Additional files}

Additional file 1: Data on the subjects included in the study. List of

subjects included in the microarray and validation studies with

information relative to their gender, age and PASI score. M, male;

F, female; PASI, Psoriasis Area Severity Index.

Additional file 2: Differentially expressed genes in in vitro activated T cells. Complete list of genes with a Benjamini and Hochberg corrected $p$-value $<0.1$ and absolute $\mathrm{FCH}>1.2$. Genes are classified in up-regulated or down-regulated and sorted by their statistical significance.

Additional file 3: Gene coexpression networks in in vitro activated T cells. 20 gene coexpression modules that resulted from weighted correlation network analysis applied to the complete microarray samples.

Additional file 4: Association results of the gene coexpression modules with different clinical and epidemiological variables. For binary variables the $t$-test was used, while for quantitative variables a linear regression approach was used. Significant $p$-values $(P<0.05)$ are highlighted in bold.

Additional file 5: Visualization of the gene modules associated with psoriasis. Using a heatmap plot we can visualize the level of adjacency of the genes that conform the four gene expression networks associated with psoriasis (light colors indicate low adjacency, dark colors high adjacency). Each module is depicted by a different color: M1 (grey), M2 (green), M3 (blue) and M4 (pink).

Additional file 6: Visualization of the eigengene network with each clinical variable. Using a heatmap plot we can visualize the relationships among the modules (representetd by their eigengenes) and each of the clinical traits. The level of correlation is a scale that goes from 0 (green) to 1 (red). Each module is depicted by a different color: grey (M1), greenyellow (M2), royalblue (M3), pink (M4), black (M5), blue (M6), brown (M7), cyan (M8), green (M9), lightcyan (M10), lightgreen (M11), lightyellow (M12), magenta (M13), midnightblue (M14), purple (M15), red (M16), salmon (M17), tan (M18), turquoise (M19), yellow (M20).

Additional file 7: Summary of enriched GO terms in SPATS2L and KLF6 coexpression networks. List of enriched $\mathrm{GO}$ terms related to biological process, cellular component and molecular function in the SPATS2L and KLF6 coexpression networks. For each term, the number of genes present in the network (count), the percentage of enrichment (\%), the associated p-value and the genes present in the network are indicated.

Additional file 8: List of GEO profiles showing highly significant differential expression of SPATS2L and KLF6 genes. Selection of SPATS2L and KLF6 GEO profiles showing highly significant differential expression. For each GEO profile, the accession number, the title and a description of their corresponding GEO DataSets are provided.

Additional file 9: List of PubMed articles on genes from the T cell gene networks associated with psoriasis risk.

\section{Competing interests}

The authors declare that they have no competing interests.

\section{Authors' contributions}

Conceived and designed the experiment: NP, AJ, CF, LP, SM. Performed the experiments: NP, CF, LP, RT. Analyzed the data: AJ, NP. Provided material: CF, $L P, E F o, E F e, M L L$. Wrote the manuscript: NP, CF, LP, RT, AJ, SM. All authors read and approved the manuscript.

\section{Acknowledgements}

We thank Devin Absher and the HudsonAlpha Institute for Biotechnology for their support. We thank Natalia Figueras Sanchez for technical assistance. This study was funded by of the Spanish Ministry of Economy and Competitiveness, grant numbers: PSE-010000-2006-6 and IPT-010000-2010-36. The study sponsor had no role in the collection, analysis or interpretation of the data.

\section{Author details}

'Rheumatology Research Group, Vall d'Hebron Research Institute, Barcelona 08035, Spain. ${ }^{2}$ Dermatology Service, Hospital Universitari Germans Trias Pujol, Badalona 08916, Spain. ${ }^{3}$ Dermatology Service, Hospital de la Santa Creu i Sant Pau, Barcelona 08040, Spain. ${ }^{4}$ Dermatology Service, Hospital Abente y Lago, La Coruña 15001, Spain. ${ }^{5}$ Dermatology Service, Hospital Universitario de Salamanca, Salamanca 37007, Spain.

Received: 17 June 2013 Accepted: 19 November 2013

Published: 23 November 2013

\section{References}

1. Enamandram M, Kimball AB: Psoriasis epidemiology: the interplay of genes and the environment. J Invest Dermatol 2013, 133(2):287-289.

2. Ferenczi K, Burack L, Pope M, Krueger JG, Austin LM: CD69, HLA-DR and the IL-2R identify persistently activated T cells in psoriasis vulgaris lesional skin: blood and skin comparisons by flow cytometry. J Autoimmun 2000, 14(1):63-78.

3. Griffiths $C E$, Barker JN: Pathogenesis and clinical features of psoriasis. Lancet 2007, 370(9583):263-271.

4. Mueller W, Herrmann B: Cyclosporin A for psoriasis. N Engl J Med 1979, 301(10):555.

5. Wrone-Smith T, Nickoloff BJ: Dermal injection of immunocytes induces psoriasis. J Clin Invest 1996, 98(8):1878-1887.

6. Abrams JR, Kelley SL, Hayes E, Kikuchi T, Brown MJ, Kang S, Lebwohl MG, Guzzo CA, Jegasothy BV, Linsley PS, et al: Blockade of T lymphocyte costimulation with cytotoxic T lymphocyte-associated antigen 4-immunoglobulin (CTLA4lg) reverses the cellular pathology of psoriatic plaques, including the activation of keratinocytes, dendritic cells, and endothelial cells. J Exp Med 2000, 192(5):681-694. 
7. Gottlieb AB, Lebwohl M, Shirin S, Sherr A, Gilleaudeau P, Singer G, Solodkina G, Grossman R, Gisoldi E, Phillips S, et al: Anti-CD4 monoclonal antibody treatment of moderate to severe psoriasis vulgaris: results of a pilot, multicenter, multiple-dose, placebo-controlled study. J Am Acad Dermatol 2000, 43(4):595-604.

8. Gottlieb SL, Gilleaudeau P, Johnson R, Estes L, Woodworth TG, Gottlieb AB, Krueger JG: Response of psoriasis to a lymphocyte-selective toxin (DAB389IL-2) suggests a primary immune, but not keratinocyte, pathogenic basis. Nat Med 1995, 1(5):442-447.

9. Nickoloff BJ: Skin innate immune system in psoriasis: friend or foe? J Clin Invest 1999, 104(9):1161-1164.

10. Prinz J, Braun-Falco O, Meurer M, Daddona P, Reiter C, Rieber P, Riethmuller $\mathrm{G}$ : Chimaeric CD4 monoclonal antibody in treatment of generalised pustular psoriasis. Lancet 1991, 338(8762):320-321.

11. Uyemura K, Yamamura M, Fivenson DF, Modlin RL, Nickoloff BJ: The cytokine network in lesional and lesion-free psoriatic skin is characterized by a T-helper type 1 cell-mediated response. J Invest Dermatol 1993, 101(5):701-705.

12. Duhen T, Geiger R, Jarrossay D, Lanzavecchia A, Sallusto F: Production of interleukin 22 but not interleukin 17 by a subset of human skin-homing memory T cells. Nat Immunol 2009, 10(8):857-863.

13. Lowes MA, Kikuchi T, Fuentes-Duculan J, Cardinale I, Zaba LC, Haider AS, Bowman EP, Krueger JG: Psoriasis vulgaris lesions contain discrete populations of Th1 and Th17 T cells. J Invest Dermatol 2008, 128(5):1207-1211.

14. Van Belle AB, de Heusch M, Lemaire MM, Hendrickx E, Warnier G, DunussiJoannopoulos K, Fouser LA, Renauld JC, Dumoutier L: IL-22 is required for imiquimod-induced psoriasiform skin inflammation in mice. J Immunol 2012, 188(1):462-469.

15. Bigler J, Rand HA, Kerkof K, Timour M, Russell CB: Cross-study homogeneity of psoriasis gene expression in skin across a large expression range. PLOS One 2013, 8(1):e52242.

16. Bowcock AM, Shannon W, Du F, Duncan J, Cao K, Aftergut K, Catier J, Fernandez-Vina MA, Menter A: Insights into psoriasis and other inflammatory diseases from large-scale gene expression studies. Hum Mol Genet 2001, 10(17):1793-1805.

17. Coda $A B$, Icen M, Smith JR, Sinha AA: Global transcriptional analysis of psoriatic skin and blood confirms known disease-associated pathways and highlights novel genomic "hot spots" for differentially expressed genes. Genomics 2012, 100(1):18-26.

18. Gudjonsson JE, Ding J, Johnston A, Tejasvi T, Guzman AM, Nair RP, Voorhees $J$ J, Abecasis GR, Elder JT: Assessment of the psoriatic transcriptome in a large sample: additional regulated genes and comparisons with in vitro models. J Invest Dermatol 2010, 130(7):1829-1840.

19. Oestreicher JL, Walters IB, Kikuchi T, Gilleaudeau P, Surette J, Schwertschlag U, Dorner AJ, Krueger JG, Trepicchio WL: Molecular classification of psoriasis disease-associated genes through pharmacogenomic expression profiling. Pharmacogenomics J 2001, 1(4):272-287.

20. Yao Y, Richman L, Morehouse C, de los Reyes M, Higgs BW, Boutrin A, White B, Coyle A, Krueger J, Kiener PA, et al: Type I interferon: potential therapeutic target for psoriasis? PLoS One 2008, 3(7):e2737.

21. Zhou A, Scoggin S, Gaynor RB, Williams NS: Identification of NF-kappa B-regulated genes induced by TNFalpha utilizing expression profiling and RNA interference. Oncogene 2003, 22(13):2054-2064

22. Jung M, Sabat R, Kratzschmar J, Seidel H, Wolk K, Schonbein C, Schutt S, Friedrich M, Docke WD, Asadullah K, et al: Expression profiling of IL-10-regulated genes in human monocytes and peripheral blood mononuclear cells from psoriatic patients during IL-10 therapy. Eur J Immunol 2004, 34(2):481-493.

23. Koczan D, Guthke R, Thiesen HJ, Ibrahim SM, Kundt G, Krentz H, Gross G, Kunz M: Gene expression profiling of peripheral blood mononuclear leukocytes from psoriasis patients identifies new immune regulatory molecules. Eur J Dermatol 2005, 15(4):251-257.

24. Lee SK, Jeon EK, Kim YJ, Seo SH, Kim CD, Lim JS, Lee JH: A global gene expression analysis of the peripheral blood mononuclear cells reveals the gene expression signature in psoriasis. Ann Dermatol 2009, 21(3):237-242.

25. Mesko B, Poliska S, Nagy L: Gene expression profiles in peripheral blood for the diagnosis of autoimmune diseases. Trends Mol Med 2011, 17(4):223-233.

26. Cotner T, Williams JM, Christenson L, Shapiro HM, Strom TB, Strominger J: Simultaneous flow cytometric analysis of human $T$ cell activation antigen expression and DNA content. J Exp Med 1983, 157(2):461-472.

27. Feng $X$, Wang $H$, Takata $H$, Day $T J$, Willen J, Hu H: Transcription factor Foxp1 exerts essential cell-intrinsic regulation of the quiescence of naive T cells. Nat Immunol 2011, 12(6):544-550.
28. Langfelder $P$, Horvath S: WGCNA: an R package for weighted correlation network analysis. BMC Bioinforma 2008, 9:559.

29. da Huang W, Sherman BT, Lempicki RA: Systematic and integrative analysis of large gene lists using DAVID bioinformatics resources. Nat Protoc 2009, 4(1):44-57.

30. Subramanian A, Tamayo P, Mootha VK, Mukherjee S, Ebert BL, Gillette MA, Paulovich A, Pomeroy SL, Golub TR, Lander ES, et al: Gene set enrichment analysis: a knowledge-based approach for interpreting genome-wide expression profiles. Proc Natl Acad Sci USA 2005, 102(43):15545-15550.

31. Mootha VK, Lindgren CM, Eriksson KF, Subramanian A, Sihag S, Lehar J, Puigserver P, Carlsson E, Ridderstrale M, Laurila E, et al: PGC-1alpharesponsive genes involved in oxidative phosphorylation are coordinately downregulated in human diabetes. Nat Genet 2003, 34(3):267-273.

32. Matthews L, Gopinath $G$, Gillespie M, Caudy M, Croft D, de Bono B, Garapati $P$, Hemish J, Hermjakob $H$, Jassal B, et al: Reactome knowledgebase of human biological pathways and processes. Nucleic Acids Res 2009, 37(Database issue):D619-D622.

33. Zhu CH, Kim J, Shay JW, Wright WE: SGNP: an essential Stress Granule/ Nucleolar Protein potentially involved in 5.8s rRNA processing/transport. PLoS One 2008, 3(11):e3716.

34. Donati G, Montanaro L, Derenzini M: Ribosome biogenesis and control of cell proliferation: p53 is not alone. Cancer Res 2012, 72(7):1602-1607.

35. Orr SJ, Boutz DR, Wang R, Chronis C, Lea NC, Thayaparan T, Hamilton E, Milewicz H, Blanc E, Mufti GJ, et al: Proteomic and protein interaction network analysis of human T lymphocytes during cell-cycle entry. $\mathrm{Mol}$ Syst Biol 2012, 8:573

36. Gutierrez A Jr, Tschumper RC, Wu X, Shanafelt TD, Eckel-Passow J, Huddleston PM 3rd, Slager SL, Kay NE, Jelinek DF: LEF-1 is a prosurvival factor in chronic lymphocytic leukemia and is expressed in the preleukemic state of monoclonal B-cell lymphocytosis. Blood 2010, 116(16):2975-2983.

37. Jeannet R, Mastio J, Macias-Garcia A, Oravecz A, Ashworth T, Geimer Le Lay AS, Jost B, Le Gras S, Ghysdael J, Gridley T, et al: Oncogenic activation of the Notch1 gene by deletion of its promoter in Ikaros-deficient T-ALL. Blood 2010, 116(25):5443-5454

38. Yang JJ, Cheng C, Yang W, Pei D, Cao X, Fan Y, Pounds SB, Neale G, Trevino $L R$, French $D$, et al: Genome-wide interrogation of germline genetic variation associated with treatment response in childhood acute lymphoblastic leukemia. Jama 2009, 301(4):393-403.

39. Brauchli YB, Jick SS, Miret M, Meier CR: Psoriasis and risk of incident cancer: an inception cohort study with a nested case-control analysis. J Invest Dermatol 2009, 129(11):2604-2612.

40. Skibola CF, Bracci PM, Halperin E, Conde L, Craig DW, Agana L, Iyadurai K, Becker N, Brooks-Wilson A, Curry JD, et al: Genetic variants at 6p21.33 are associated with susceptibility to follicular lymphoma. Nat Genet 2009, 41(8):873-875

41. Cho-Vega JH, Medeiros LJ, Prieto VG, Vega F: Leukemia cutis. Am J Clin Pathol 2008, 129(1):130-142.

42. Andreoli V, Gehrau RC, Bocco JL: Biology of Kruppel-like factor 6 transcriptional regulator in cell life and death. IUBMB Life 2010, 62(12):896-905.

43. Cao Z, Sun X, Icli B, Wara AK, Feinberg MW: Role of Kruppel-like factors in leukocyte development, function, and disease. Blood 2010, 116(22):4404-4414.

44. Yang XO, Doty RT, Hicks JS, Willerford DM: Regulation of T-cell receptor D beta 1 promoter by KLF5 through reiterated GC-rich motifs. Blood 2003, 101(11):4492-4499.

45. Warke VG, Nambiar MP, Krishnan S, Tenbrock K, Geller DA, Koritschoner NP Atkins JL, Farber DL, Tsokos GC: Transcriptional activation of the human inducible nitric-oxide synthase promoter by Kruppel-like factor 6 . $J$ Biol Chem 2003, 278(17):14812-14819.

46. Kiaii S, Kokhaei P, Mozaffari F, Rossmann E, Pak F, Moshfegh A, Palma M, Hansson L, Mashayekhi K, Hojjat-Farsangi M, et al: T cells from indolent CLL patients prevent apoptosis of leukemic $B$ cells in vitro and have altered gene expression profile. Cancer Immunol Immunother 2013, 62(1):51-63.

47. Bensinger SJ, Bradley MN, Joseph SB, Zelcer N, Janssen EM, Hausner MA, Shih R, Parks JS, Edwards PA, Jamieson BD, et al: LXR signaling couples sterol metabolism to proliferation in the acquired immune response. Cell 2008, 134(1):97-111.

48. Bensinger SJ, Tontonoz P: Integration of metabolism and inflammation by lipid-activated nuclear receptors. Nature 2008, 454(7203):470-477.

49. Marelli-Berg FM, Fu H, Mauro C: Molecular mechanisms of metabolic reprogramming in proliferating cells: implications for T-cell-mediated immunity. Immunology 2012, 136(4):363-369. 
50. Pearce EL: Metabolism in T cell activation and differentiation. Curr Opin Immunol 2010, 22(3):314-320.

51. Krishnan N, Fu C, Pappin DJ, Tonks NK: H2S-Induced sulfhydration of the phosphatase PTP1B and its role in the endoplasmic reticulum stress response. Sci Signal 2011, 4(203):ra86.

52. Miller TW, Wang EA, Gould S, Stein EV, Kaur S, Lim L, Amarnath S, Fowler DH, Roberts DD: Hydrogen sulfide is an endogenous potentiator of T cell activation. J Biol Chem 2012, 287(6):4211-4221.

53. Mungrue IN, Pagnon J, Kohannim O, Gargalovic PS, Lusis AJ: CHAC1/ MGC4504 is a novel proapoptotic component of the unfolded protein response, downstream of the ATF4-ATF3-CHOP cascade. J Immunol 2009, 182(1):466-476.

54. Venter JC, Adams MD, Myers EW, Li PW, Mural RJ, Sutton GG, Smith HO, Yandell M, Evans CA, Holt RA, et al: The sequence of the human genome. Science 2001, 291(5507):1304-1351.

55. Chakrabarti A, Chen AW, Varner JD: A review of the mammalian unfolded protein response. Biotechnol Bioeng 2011, 108(12):2777-2793.

56. Hasnain SZ, Lourie R, Das I, Chen AC, McGuckin MA: The interplay between endoplasmic reticulum stress and inflammation. Immunol Cell Biol 2012, 90(3):260-270

57. Park H, Bourla AB, Kastner DL, Colbert RA, Siegel RM: Lighting the fires within: the cell biology of autoinflammatory diseases. Nat Rev Immunol 2012, 12(8):570-580.

58. Nestle FO, Conrad C, Tun-Kyi A, Homey B, Gombert M, Boyman O, Burg G, Liu YJ, Gilliet M: Plasmacytoid predendritic cells initiate psoriasis through interferon-alpha production. J Exp Med 2005, 202(1):135-143.

59. Tohyama M, Yang L, Hanakawa Y, Dai X, Shirakata Y, Sayama K: IFN-alpha enhances IL-22 receptor expression in keratinocytes: a possible role in the development of psoriasis. J Invest Dermatol 2012, 132(7):1933-1935.

60. Yoneyama $M$, Kikuchi $M$, Natsukawa $T$, Shinobu $N$, Imaizumi T, Miyagishi $M$, Taira K, Akira S, Fujita T: The RNA helicase RIG-I has an essential function in double-stranded RNA-induced innate antiviral responses. Nat Immunol 2004, 5(7):730-737.

61. Shaw MH, Reimer T, Sanchez-Valdepenas C, Warner N, Kim YG, Fresno M, Nunez G: T cell-intrinsic role of Nod2 in promoting type 1 immunity to Toxoplasma gondii. Nat Immunol 2009, 10(12):1267-1274.

62. Wolk K, Witte K, Witte E, Raftery M, Kokolakis G, Philipp S, Schonrich G Warszawska K, Kirsch S, Prosch S, et al: IL-29 Is Produced by TH17 Cells and Mediates the Cutaneous Antiviral Competence in Psoriasis. Sci Trans/ Med 2013, 5(204):204ra129.

63. Team RDC: R: A Language and Environment for Statistical Computing. Vienna: R Foundation for Statistical Computing; 2009.

64. Bolstad BM, Irizarry RA, Astrand M, Speed TP: A comparison of normalization methods for high density oligonucleotide array data based on variance and bias. Bioinformatics 2003, 19(2):185-193.

65. Gentleman RC, Carey VJ, Bates DM, Bolstad B, Dettling M, Dudoit S, Ellis B, Gautier L, Ge Y, Gentry J, et al: Bioconductor: open software development for computational biology and bioinformatics. Genome Biol 2004, 5(10):R80.

66. Benjamini YHM: Controlling the false discovery rate: a practical and powerful approach to multiple testing. J R Stat Soc 1995, 57(1):289-300.

67. Allen JD, Xie Y, Chen M, Girard L, Xiao G: Comparing statistical methods for constructing large scale gene networks. PLoS One 2012, 7(1):e29348.

68. Zhang B, Horvath S: A general framework for weighted gene coexpression network analysis. Stat Appl Genet Mol Biol 2005, 4(1):1544-6115.

69. Langfelder $\mathrm{P}$, Zhang $\mathrm{B}$, Horvath $\mathrm{S}$ : Defining clusters from a hierarchical cluster tree: the Dynamic Tree Cut package for R. Bioinformatics 2008, 24(5):719-720.

doi:10.1186/1471-2164-14-825

Cite this article as: Palau et al:: Genome-wide transcriptional analysis of $T$ cell activation reveals differential gene expression associated with psoriasis. BMC Genomics 2013 14:825.

\section{Submit your next manuscript to BioMed Central and take full advantage of:}

- Convenient online submission

- Thorough peer review

- No space constraints or color figure charges

- Immediate publication on acceptance

- Inclusion in PubMed, CAS, Scopus and Google Scholar

- Research which is freely available for redistribution

Submit your manuscript at www.biomedcentral.com/submit
Ciomed Central 